\title{
Backstepping control of a wave PDE with unstable source terms and dynamic boundary
}

\author{
Christophe Roman ${ }^{1}$, Delphine Bresch-Pietri ${ }^{2}$, Eduardo Cerpa ${ }^{3}$, Christophe Prieur $^{1}$ and Olivier Sename ${ }^{1}$
}

\begin{abstract}
This paper presents the design of an exponentially stabilizing controller for a one-dimensional wave partial differential equation (PDE). The control is acting on a Robin's boundary condition while the opposite boundary satisfies an unstable dynamic. The wave is also subject to unstable in-domain source terms. Closed-loop exponential stabilization is obtained via a full-state backstepping controller. The existence and uniqueness of this backstepping transformation is proven, using the method of successive approximations.
\end{abstract}

\section{INTRODUCTION}

We investigate the feedback control problem of the following system

$$
\begin{aligned}
& u_{t t}(x, t)=u_{x x}(x, t)+\lambda u_{t}(x, t)+\beta u(x, t) \\
& u_{x}(1, t)=a_{1} u_{t}(1, t)+a_{2} u(1, t)+U(t) \\
& u_{t t}(0, t)=b_{1} u_{t}(0, t)+b_{2} u_{x}(0, t)+b_{3} u(0, t)
\end{aligned}
$$

in which $U$ is a scalar control input. The scalar values $a_{1}$, and $a_{2}$ are coefficients of the controlled boundary, $\lambda$ is an in-domain velocity source term, $\beta$ is an in-domain position source term, and the dynamical boundary coefficients are $b_{1}$, $b_{2} \neq 0$, and $b_{3}$. All parameters are supposed to be known.

The main specificity of (1)-(3) consists in its boundary condition (3) (for information about wave PDEs and corresponding boundary conditions, see [5] and p.30 in particular). This second-order dynamical boundary condition naturally arises when applying Newton second law of motion. Yet, it has seldom been considered in the literature. More specifically, one can refer to [6] for a study of a one-dimensional wave equation subject to a Wentzell boundary condition. System (1)(3) can indeed be reformulated as such, replacing the derivative $u_{t t}$ in (3) with the help of (1). One can also refer to [4], [10] for higher order systems. However, this framework is not state-of-the-art and the stabilization of (1)-(3) remains an open problem.

In the present paper, the proposed control design is based on backstepping. Note that there exists a fair amount of alternative control techniques. Indeed, most of the finite dimensional control tools have been extended to infinite dimensional systems through the theory of semigroup, e.g. frequency based control, LQR (see [2] for an introduction on semigroup theory and

\footnotetext{
1 Univ. Grenoble Alpes, CNRS, Grenoble-INP, GIPSA-lab, F-38000, Grenoble, France (name.surmane@gipsa-lab.fr)

2 MINES ParisTech, PSL Research University CAS - Centre Automatique et Systèmes, 60 bd Saint Michel, 75006 Paris, FRANCE (delphine.bresch-pietri@mines-paristech.fr)

${ }^{3}$ Eduardo Cerpa is with Departemento de Matemática, Universidad Técnica Federico Santa Maria, Casilla 110-V, Valparaiso, Chile (eduardo.cerpa@usm.cl)

Acknowledgment: This work has been partially supported by ANR-15CE23-0008 MACS-DRILL, ECOS-CONICYT project C16E06 and Basal Project FB0008-AC3E.
}

semigroup based-control techniques). Among other control strategies (see for example [9], in which the flatness-based tracking of a pure wave $\operatorname{PDE}(\lambda=\beta=0)$ with two secondorder dynamic boundary conditions is presented) backstepping is acknowledged for its relative simplicity for practitioners (see the monograph [7] which presents the main concepts of backstepping for infinite dimensional systems). It has already been applied in the literature in related yet different contexts. For example, we refer the reader to [14] for a treatment of a wave PDE with space-dependent in-domain velocity and position source terms $(\lambda(x), \beta(x))$ associated with Dirichlet's boundary conditions (see references therein for further references about the control of the wave equation). Also, in [16], a pure wave $\operatorname{PDE}(\lambda=\beta=0)$ with a first-order dynamic anti-damped boundary condition is considered. The controller and the boundary observer have both been designed using backstepping.

The framework of coupled PDE-ODEs can also be considered in order to deal with dynamic boundary conditions. For example in [13], the authors express the system (1)-(3) (with $\beta=b_{3}=a_{1}=a_{2}=0$ ) as a wave PDE with Dirichlet's boundary conditions coupled with an ODE (Ordinary Differential Equation) to perform backstepping. But they need an additional assumption on the state space regularity. Another approach is to consider coupled hyperbolic PDE-ODE, as done in [8]. However, the reformulation of the wave PDE into the former framework, using Riemann invariants, may not be straightforward since it involves the position terms $(u(., t))$. Note that there seems to be no problem if $\beta=a_{2}=b_{3}=0$.

For these reasons, we propose a more direct approach. As far as we know, there is no design stabilizing (1)-(3).

Our first objective is to improve our control design from [12], by allowing a change of in-domain viscous damping between the original system and the target system and thus giving an additional degree of freedom to the designer. Futhermore, in [12], the system under consideration does not involve any position source terms (case where $\beta=b_{3}=a_{1}=a_{2}=0$ ). As a result, the control problem considered here is different: in [12], the system is exponentially stabilized toward an attractor (by considering a functional of $\left\|u_{t}\right\|_{L_{2}},\left\|u_{x}\right\|_{L_{2}}$, and $u_{t}(0, t)^{2}$ ), and in the present paper the system is stabilized toward the origin.

The main contribution of this paper is the design of a backstepping control law which exponentially stabilizes the system (1)-(3). Standardly, we use a Volterra transformation mapping the original system into a stable target system and prove existence and uniqueness of this transformation using the method of successive approximations. The originality of our approach lies in our methodology to handle the dynamic boundary conditions, both in the establishment of the kernel 
integral equation and the method of successive approximations.

The paper is organized as follows. First, in Section II, the control law is defined and the corresponding stability result is stated. Then, Section III is dedicated to the proof of the stability result. Finally, we draw some perspectives for future works in Section IV.

Notation In this paper, we consider the following norms

$$
\begin{aligned}
\|u\|_{L_{2}}^{2} & :=\int_{0}^{1} u(x)^{2} d x,\|u\|_{H_{1}}^{2}:=\|u\|_{L_{2}}^{2}+\int_{0}^{1} u_{x}(x)^{2} d x \\
|\Omega(.)|_{\infty} & :=\sup _{i=1 \ldots n}\left\{\left|\Omega_{i}(.)\right|\right\},\|\Omega\|_{L_{\infty}}:=\sup _{\substack{i=1 \ldots n \\
(x, y) \in[0,1]^{2}}}\left\{\left|\Omega_{i}(x, y)\right|\right\}
\end{aligned}
$$

where $\Omega$ is a vector of $n$ elements $\Omega_{i} \in L_{\infty}\left([0,1]^{2} ; \mathbb{R}\right)$. For a function $k:(x, y) \mapsto k(x, y), k^{\prime}(x, x)$ is used to denote the total derivative of $k$ evaluated at $(x, x)$, i.e.,

$$
k^{\prime}(x, x):=\left.\frac{\partial k}{\partial x}\right|_{(x, x)}+\left.\frac{\partial k}{\partial y}\right|_{(x, x)}
$$

\section{BACKSTEPPING STATE FEEDBACK CONTROL}

The objective of this section is to present the backstepping transformation that maps (1)-(3), along with its corresponding feedback control law, into an exponentially stable target system. This target system is chosen as

$$
\begin{aligned}
& w_{t t}(x, t)=w_{x x}(x, t)-\lambda_{w} w_{t}(x, t)-\beta_{w} w(x, t) \\
& w_{x}(1, t)=-c_{1} w_{t}(1, t)-c_{2} w(1, t) \\
& w_{t t}(0, t)=-d_{1} w_{t}(0, t)+d_{2} w_{x}(0, t)-d_{3} w(0, t)
\end{aligned}
$$

in which the boundary parameters $c_{1}, c_{2}, d_{1}, d_{2}$, and $d_{3}$ are positive constants. $\lambda_{w}>0$ is an in-domain velocity source term and $\beta_{w}>0$ is an in-domain position source term. As all these parameters are positive, this target system is exponentially stable (see Lemma 3 in Section III-D).

To solve the previously introduced control objective, the following backstepping transformation is selected

$$
w(x, t)=u(x, t)-\int_{0}^{x} K^{T}(x, y) \chi(y, t) d y-\gamma(x) u_{t}(0, t)
$$

in which $K$ and $\chi$ are defined as

$$
K(x, y):=\left[\begin{array}{c}
k(x, y) \\
s(x, y) \\
m(x, y)
\end{array}\right], \quad \chi(x, t):=\left[\begin{array}{c}
u(x, t) \\
u_{t}(x, t) \\
u_{x}(x, t)
\end{array}\right]
$$

and the kernel $(K, \gamma)$ is chosen as the unique solution (see Lemma 2, Section III-B) of the following system

$$
\begin{aligned}
K_{x x}(x, y)-K_{y y}(x, y) & =B_{K} K_{y}(x, y)+C_{K} K(x, y) \\
K^{\prime}(x, x) & =D_{K} K(x, x)+E_{K} \\
{\left[\begin{array}{c}
k_{y}(x, 0) \\
m_{y}(x, 0)
\end{array}\right] } & =H_{K} K(x, 0) \\
s_{x x}(x, 0) & =b_{2} s_{y}(x, 0)+H_{S} K(x, 0) \\
{\left[\begin{array}{c}
K(0,0) \\
s_{x}(0,0)
\end{array}\right] } & =J_{K} \\
\gamma(x) & =\frac{s(x, 0)}{b_{2}}
\end{aligned}
$$

in which

$$
B_{K}^{T}:=-\left(\Theta_{2} \Theta_{1}+\Theta_{1} \Theta_{2}+\lambda_{w} \Theta_{2}\right)
$$

$$
\begin{aligned}
& C_{K}^{T}:=\Theta_{1}^{2}+\lambda_{w} \Theta_{1}+\beta_{w} \mathrm{I}_{3} \\
& D_{K}^{T}:=\frac{1}{2}\left(\Theta_{2} \Theta_{1}+\Theta_{1} \Theta_{2}+\lambda_{w} \Theta_{2}\right) \\
& E_{K}^{T}:=-\frac{1}{2}\left[\begin{array}{lll}
\beta+\beta_{w} & \lambda+\lambda_{w} & 0
\end{array}\right] \\
& H_{K}:=\left[\begin{array}{ccc}
\lambda_{w} & \beta-\left(\lambda_{w}+b_{1}\right) \frac{b_{3}}{b 2} & b_{3} \\
1 & \lambda-b_{1} & b_{2}
\end{array}\right] \\
& H_{S}:=\left[\begin{array}{lll}
0 & \beta_{w}+b_{3}+\lambda_{w} b_{1}+b_{1}^{2} & -\left(b_{2} \lambda_{w}+b_{2} b_{1}\right)
\end{array}\right]
\end{aligned}
$$

where

$$
\begin{gathered}
\Theta_{1}:=\left[\begin{array}{ccc}
0 & 1 & -1 \\
\beta & \lambda & 0 \\
0 & 0 & 0
\end{array}\right], \quad \Theta_{2}:=\left[\begin{array}{lll}
1 & 0 & 0 \\
0 & 0 & 1 \\
0 & 1 & 0
\end{array}\right] \\
J_{K}:=-\frac{1}{d_{2}}\left[\begin{array}{c}
d_{3}+b_{3} \\
0 \\
b_{2}-d_{2} \\
\left(b_{1}+d_{1}\right) b_{2}
\end{array}\right]
\end{gathered}
$$

The construction of (9) is inspired by [13] and by [16] for the integral terms. However, the kernel equations (11)(16) are different from both papers. Indeed, (11) presents an additional source term which reveals troublesome to handle in the subsequent analysis. Moreover, note that the boundary condition (13) is similar to the one of [15]. However, the boundary condition in [15] is scalar, and contains in addition an integral term. The considered boundary (13) is, on the one hand, simpler as there is no integral term, but, on the other hand, it is a vectorial one coupled with another boundary condition. Yet, the recursive assumption we use in the method of successive approximations (Section III-B) is different from [15] and new, up to our knowledge.

Finally, the corresponding control law is

$$
\begin{aligned}
& U(t):=K^{T}(1,1) \chi(1, t)+\int_{0}^{1} K_{x}^{T}(1, y) \chi(y, t) d y+\gamma^{\prime}(1) u_{t}(0, t) \\
& -\left(a_{1}+c_{1}\right) u_{t}(1, t)-\left(a_{2}+c_{2}\right) u(1, t)+c_{2}\left[\gamma(1) u_{t}(0, t)\right. \\
& \left.+\int_{0}^{1} K^{T}(1, y) \chi(y, t) d y\right]+c_{1}\left[\left[K^{T}(1, y) \Theta_{2} \chi(y, t)\right]_{y=0}^{1}\right. \\
& \left.+\int_{0}^{1}\left[K^{T} \Theta_{1}-K_{y}^{T} \Theta_{2}\right](1, y) \chi(y, t) d y+\gamma(1) \Theta_{3} \chi(0, t)\right]
\end{aligned}
$$

in which

$$
\Theta_{3}:=\left[\begin{array}{lll}
b_{3} & b_{1} & b_{2}
\end{array}\right]
$$

Theorem 1: Consider the closed-loop system consisting of the plant (1)-(3) with $b_{2} \neq 0$, together with the control law (25) in which the kernel $(K, \gamma)$ is the solution of (11)-(16). Define the functional (the $L_{2}$ and $H_{1}$ norms are defined in (4))

$$
\Gamma\left(u, u_{t}\right):=\|u\|_{H_{1}}^{2}+\left\|u_{t}\right\|_{L_{2}}^{2}+u(1, t)^{2}+u(0, t)^{2}+u_{t}(0, t)^{2}
$$

There exist $\rho>0$ and $R>0$ such that

$$
\Gamma\left(u(t), u_{t}(t)\right) \leqslant R \Gamma\left(u(0), u_{t}(0)\right) e^{-\rho t}, \quad t \geqslant 0
$$

i.e., the closed-loop system is exponentially stable.

Note that the control law $U(25)$ is expressed in terms of $u$, $u_{t}$ and $u_{x}$ but can also be written, using integration by parts, in terms of $u$ and $u_{t}$ only. Yet, it requires full-state measurement, which is seldom the case in practice. Future work will focus on relieving this constraint. 


\section{PROOF OF THEOREM 1}

First, Section III-A presents the derivation of the backstepping kernel equations (11)-(16). Second, the existence and uniqueness of a solution to these equations is proven in Section III-B. In Section III-C, we analyze the invertibility of the backstepping transformation. The proof of the target system stability is established in Section III-D, through a Lyapunov analysis. Then, in Section III-E, the equivalence between the target system Lyapunov functional and the functional $\Gamma$ defined in (27) is established. Finally, using all previous developments, we conclude the proof of Theorem 1 in Section III-F.

\section{A. Backstepping transformation}

This section is devoted to the establishment of (11)-(16). Before stating the corresponding lemma, consider the vector $\chi(x, t)$ defined in (10). Using the system equation (1) together with the boundary condition (3), it is straightforward to show that

$$
\begin{aligned}
\chi_{t}(x, t) & =\Theta_{1} \chi(x, t)+\Theta_{2} \chi_{x}(x, t) \\
u_{t t}(0, t) & =\Theta_{3} \chi(0, t) \\
\chi_{t}(0, t) & =\Theta_{4} \underline{\chi}(0, t) \\
\chi(0, t) & =\Theta_{5} \underline{\chi}(0, t)
\end{aligned}
$$

where $\Theta_{1}, \Theta_{2}$, and $\Theta_{3}$ are defined in (23) and (26), and with

$$
\begin{aligned}
\underline{\chi}(0, t) & :=\left[\begin{array}{llll}
u(0, t) & u_{t}(0, t) & u_{x}(0, t) & u_{x t}(0, t)
\end{array}\right]^{T} \\
\Theta_{4} & :=\left[\begin{array}{cccc}
0 & 1 & 0 & 0 \\
b_{3} & b_{1} & b_{2} & 0 \\
0 & 0 & 0 & 1
\end{array}\right], \Theta_{5}:=\left[\begin{array}{llll}
1 & 0 & 0 & 0 \\
0 & 1 & 0 & 0 \\
0 & 0 & 1 & 0
\end{array}\right]
\end{aligned}
$$

Note that $\Theta_{1}$ and $\Theta_{2}$ such that (29) holds are not unique. The choice made in (23) however guarantees that $\Theta_{2}^{2}=I_{3}$, a property which we use in the sequel.

Lemma 1: If $(K, \gamma)$ in (9) is solution of (11)-(16), then the backstepping transformation (9) along the control law (25) map (1)-(3) into the target system (6)-(8) .

Proof of Lemma 1: Consider the time derivative of (9)

$$
w_{t}(x, t)=u_{t}(x, t)-\int_{0}^{x} K^{T}(x, y) \chi_{t}(y, t) d y-\gamma(x) u_{t t}(0, t)
$$

Using (29), an integration by parts and (30), one expresses (35) as

$$
\begin{aligned}
w_{t}(x, t) & =u_{t}(x, t)-\left[K^{T}(x, y) \Theta_{2} \chi(y, t)\right]_{y=0}^{x} \\
& -\int_{0}^{x}\left[K^{T} \Theta_{1}-K_{y}^{T} \Theta_{2}\right](x, y) \chi(y, t) d y-\gamma(x) \Theta_{3} \chi(0, t)
\end{aligned}
$$

Consider the time derivative of (36). Using (1), (29), the fact that $\Theta_{2}^{2}=I_{3}$, and an integration by parts, one obtains

$$
\begin{aligned}
& w_{t t}(x, t)=u_{x x}(x, t)+\lambda u_{t}(x, t)+\beta u(x, t) \\
& -\left[K^{T}(x, y) \Theta_{2} \chi_{t}(y, t)+\left(K^{T}(x, y) \Theta_{1} \Theta_{2}-K_{y}^{T}(x, y)\right) \chi(y, t)\right]_{y=0}^{x} \\
& -\int_{0}^{x}\left[\left(K^{T} \Theta_{1}-K_{y}^{T} \Theta_{2}\right) \Theta_{1}-\left(K_{y}^{T} \Theta_{1} \Theta_{2}-K_{y y}^{T}\right)\right](x, y) \chi(y, t) d y \\
& -\gamma(x) \Theta_{3} \chi_{t}(0, t)
\end{aligned}
$$

Consider the first and second space derivatives of (9), which can be written as

$$
\begin{aligned}
& \begin{aligned}
& w_{x}(x, t)=u_{x}(x, t)-K^{T}(x, x) \chi(x, t)-\int_{0}^{x} K_{x}^{T}(x, y) \chi(y, t) d y \\
&-\gamma^{\prime}(x) u_{t}(0, t) \\
& w_{x x}(x, t)=u_{x x}(x, t)-\left(K^{\prime T}(x, x)+K_{x}^{T}(x, x)\right) \chi(x, t) \\
&-K^{T}(x, x) \chi_{x}(x, t)-\int_{0}^{x} K_{x x}^{T}(x, y) \chi(y, t) d y-\gamma^{\prime \prime}(x) u_{t}(0, t)
\end{aligned}
\end{aligned}
$$

Gathering (9), (36), (37), and (39) the target system equation (6) holds if the kernel $(K, \gamma)$ satisfies the following conditions

$$
\begin{aligned}
& K^{T}(x, y) \Theta_{1}^{2}-K_{y}^{T}(x, y) \Theta_{2} \Theta_{1}-K_{y}^{T}(x, y) \Theta_{1} \Theta_{2}+K_{y y}^{T}(x, y) \\
& -K_{x x}^{T}(x, y)+\lambda_{w} K^{T}(x, y) \Theta_{1}-\lambda_{w} K_{y}^{T}(x, y) \Theta_{2}+\beta_{w} K^{T}(x, y)=0 \\
& K^{T}(x, x) \Theta_{2} \chi_{t}(x, t)-\lambda \Theta_{6} \chi(x, t)-\beta \Theta_{8} \chi(x, t) \\
& +\left[K^{T}(x, x) \Theta_{1} \Theta_{2}-K_{y}^{T}(x, x)\right] \chi(x, t)-\lambda_{w} \Theta_{6} \chi(x, t) \\
& +\lambda_{w} K^{T}(x, x) \Theta_{2} \chi(x, t)-\beta_{w} \Theta_{8} \chi(x, t) \\
& -\left(K^{\prime T}(x, x)+K_{x}^{T}(x, x)\right) \chi(x, t)-K^{T}(x, x) \chi_{x}(x, t)=0 \\
& \gamma(x) \Theta_{3} \chi_{t}(0, t)+\left(-K^{T}(x, 0) \Theta_{1} \Theta_{2}+K_{y}^{T}(x, 0)\right) \chi(0, t) \\
& -K^{T}(x, 0) \Theta_{2} \chi(0, t)-\gamma^{\prime \prime}(x) \Theta_{6} \chi(0, t)-\lambda_{w} K^{T}(x, 0) \Theta_{2} \chi(0, t) \\
& +\lambda_{w} \gamma(x) \Theta_{3} \chi(0, t)+\beta_{w} \gamma(x) \Theta_{6} \chi(0, t)=0
\end{aligned}
$$

in which

$$
\Theta_{6}:=\left[\begin{array}{lll}
0 & 1 & 0
\end{array}\right], \quad \Theta_{8}:=\left[\begin{array}{lll}
1 & 0 & 0
\end{array}\right]
$$

Following the definitions (17)-(18) of $B_{K}$ and $C_{K}$, (40) can be rewritten as (11). Moreover, using (29), (41) can be reformulated as (12). Furthermore, using (31) and (32), one obtains that (42) is equivalent to the four following scalar conditions

$$
\begin{aligned}
\beta s(x, 0) & -k_{y}(x, 0)+\lambda_{w} k(x, 0)+b_{3} m(x, 0) \\
& -\lambda_{w} b_{3} \gamma(x)-b_{1} b_{3} \gamma(x)=0 \\
-\beta_{w} \gamma(x) & -s_{y}(x, 0)+\gamma^{\prime \prime}(x)+\lambda_{w} m(x, 0)-b_{3} \gamma(x) \\
& -\lambda_{w} b_{1} \gamma(x)+b_{1} m(x, 0)-b_{1}^{2} \gamma(x)=0 \\
k(x, 0) & -m_{y}(x, 0)+\lambda s(x, 0)+b_{2} m(x, 0) \\
& -\lambda_{w} b_{2} \gamma(x)-b_{1} b_{2} \gamma(x)+\lambda_{w} s(x, 0)=0 \\
s(x, 0) & -b_{2} \gamma(x)=0
\end{aligned}
$$

which can be reformulated as (13), (14), and (16).

Now, the boundary condition (8) gives one last condition on the kernel. As, from (9), (36), (37), and (38),

$$
\left\{\begin{aligned}
w_{t t}(0, t) & =\left[\Theta_{3} \Theta_{5}-\gamma(0) \Theta_{3} \Theta_{4}\right] \chi(0, t) \\
w_{t}(0, t) & =\left[\Theta_{6}-\gamma(0) \Theta_{3}\right] \Theta_{5} \underline{\chi}(0, t) \\
w_{x}(0, t) & =\left[\Theta_{7}-K^{T}(0,0)-\bar{\gamma}^{\prime}(0) \Theta_{6}\right] \Theta_{5} \underline{\chi}(0, t) \\
w(0, t) & =\left[\Theta_{8}-\gamma(0) \Theta_{6}\right] \Theta_{5} \underline{\chi}(0, t)
\end{aligned}\right.
$$

in which $\Theta_{7}:=\left[\begin{array}{lll}0 & 0 & 1\end{array}\right]$, thus, a sufficient condition for (8) to hold is

$$
\left[\begin{array}{c}
b_{3}+d_{3} \\
b_{1}+d_{1} \\
b_{2}-d_{2} \\
0
\end{array}\right]=-d_{2}\left[\begin{array}{c}
k(0,0) \\
s(0,0) \\
m(0,0) \\
0
\end{array}\right]+\gamma(0)\left[\begin{array}{c}
* \\
* \\
* \\
b_{2}
\end{array}\right]-\gamma^{\prime}(0)\left[\begin{array}{c}
0 \\
d_{2} \\
0 \\
0
\end{array}\right]
$$

which, using (47), is (15).

Finally the control law (25) can be obtained from (7) with (9), (36), (38), and using (2). 


\section{B. Existence and uniqueness of the solution of (11)-(15)}

The PDE (11)-(15) presents two different types of boundary conditions at $(x, 0)$, namely (13) and (14). The key idea in the sequel is to address each condition separately. First, consider the following change of variable

$$
G(\zeta, \eta)=e^{\frac{1}{2} B_{K}(\zeta-\eta)} K(\zeta+\eta, \zeta-\eta)
$$

The idea behind (50) is, on one hand, to gather the high order derivatives $\left(K_{x x}-K_{y y}\right)$ in (11) into one term $\left(G_{\eta \zeta}\right)$, with $x=\zeta+\eta$ and $y=\zeta-\eta$, and, on the other hand, to cancel the term in $K_{y}$ in (11), which is done with $G=e^{\frac{1}{2} B_{K} y} K$.

Consider also the following definitions

$$
\begin{gathered}
\phi_{k, m}:=\left[\begin{array}{lll}
1 & 0 & 0 \\
0 & 0 & 1
\end{array}\right], \quad \phi_{s}:=\left[\begin{array}{lll}
0 & 1 & 0
\end{array}\right] \\
K^{k, m}:=\phi_{k, m} K, G^{k, m}:=\phi_{k, m} G, K^{s}:=\phi_{s} K, G^{s}:=\phi_{s} G \\
\text { Note that } \quad G=\phi_{k, m}^{T} G^{k, m}+\phi_{s}^{T} G^{s}
\end{gathered}
$$

Proposition 1: The two following statements are equivalent

(i). $K$ is a solution of (11)-(15).

(ii). $G$ is a solution of

$$
\begin{aligned}
G_{\zeta \eta}(\zeta, \eta) & =C_{G}(\zeta, \eta) G(\zeta, \eta) \\
G(\zeta, 0) & =F_{G}(\zeta) \\
G_{\zeta}^{k, m}(\eta, \eta) & =H_{G} G(\eta, \eta)+G_{\eta}^{k, m}(\eta, \eta) \\
G^{s}(\eta, \eta) & =2 \int_{0}^{\eta} \int_{0}^{z}\left[b_{2}\left(G_{\zeta}^{s}(\mu, \mu)-G_{\eta}^{s}(\mu, \mu)\right)\right. \\
& \left.+H_{G S} G(\mu, \mu)\right] d \mu d z+J_{G S}(2 \eta)
\end{aligned}
$$

in which

$$
\begin{gathered}
C_{G}(\zeta, \eta)=e^{\frac{1}{2} B_{K}(\zeta-\eta)}\left(C_{K}-\frac{1}{4} B_{K}^{2}\right) e^{-\frac{1}{2} B_{K}(\zeta-\eta)} \\
F_{G}(\zeta)=e^{\frac{1}{2} B_{K} \zeta} F_{K}(\zeta) \\
F_{K}(\zeta)=K(x, x)=e^{D_{K} x} J_{G K}+\int_{0}^{x} e^{D_{K}(x-s)} E_{K} d s \\
H_{G}=2 H_{K}+\phi_{k, m} B_{K}, \quad H_{G S}=2 H_{S}-b_{2} \phi_{S} B_{K} \\
J_{G S}(x):=\left[\begin{array}{llll}
0 & 1 & 0 & x
\end{array}\right] J_{K}, \quad J_{G K}:=\Theta_{5} J_{K}
\end{gathered}
$$

Proof: $(i) \Rightarrow(i i)$. The establishment of (54) and (55) is straightforward. Besides, from the fact that

$$
s(x, 0)=\int_{0}^{x} \int_{0}^{s} s_{x x}(z, 0) d z d s+x s_{x}(0,0)+s(0,0)
$$

using (14), (16), (62), and $z=2 \mu$, one gets

$$
s(x, 0)=\int_{0}^{x} \int_{0}^{\frac{s}{2}}\left[2 b_{2} s_{y}(2 \mu, 0)+2 H_{S} K(2 \mu, 0)\right] d \mu d s+J_{G S}(x)
$$

From (50), it holds

$$
G_{\zeta}(\eta, \eta)-G_{\eta}(\eta, \eta)=2 K_{y}(2 \eta, 0)+B_{K} K(2 \eta, 0)
$$

Thus multiplying (65) by $\phi_{s}$ defined in (51), one writes

$$
2 s_{y}(2 \eta, 0)=G_{\zeta}^{s}(\eta, \eta)-G_{\eta}^{s}(\eta, \eta)-\phi_{s} B_{K} G(\eta, \eta)
$$

Replacing (66) into (64), for $x=2 \eta$, and $2 z=s$, one obtains

$$
\begin{aligned}
G^{s}(\eta, \eta) & =\int_{0}^{\eta} 2 \int_{0}^{z}\left[b _ { 2 } \left(G_{\zeta}^{s}(\mu, \mu)-G_{\eta}^{s}(\mu, \mu)\right.\right. \\
& \left.\left.-\phi_{s} B_{K} G(\mu, \mu)\right)+2 H_{S} G(\mu, \mu)\right] d \mu d z+J_{G S}(2 \eta)
\end{aligned}
$$


it holds

$$
\begin{aligned}
G^{k, m}(\zeta, \zeta) & =-\int_{0}^{\zeta} H_{G} G(s, s) d s+2 G^{k, m}(\zeta, 0) \\
& +2 \phi_{k, m} \int_{0}^{\zeta} \int_{0}^{s} C_{G}(s, z) G(s, z) d z d s-G^{k, m}(0,0)
\end{aligned}
$$

Finally, using (54) in (72), and using (53), it holds

$$
G(\eta, \eta)=\phi_{k, m}^{T} G^{k, m}(\eta, \eta)+\phi_{s}^{T} G^{s}(\eta, \eta)
$$

thus with (76) and (78), one gets

$$
G(\zeta, \eta)=\mathscr{F}(G(\zeta, \eta))+(\Delta G)^{0}(\zeta, \eta)
$$

in which $(\Delta G)^{0}(\zeta, \eta)$ and $\mathscr{F}($.$) are defined in (70) and (71).$

Now, we use a standard iterative method to prove that (80) has a unique solution. The initial guess is $(\Delta G)^{0}$ defined in (70), and initiates the induction (69) for $n \in \mathbb{N}$. Define $M \in \mathbb{R}^{+}$ as an upper bound of $(\Delta G)^{0}$. By the definition of $|\cdot|_{\infty}$ and $\|\cdot\|_{L_{\infty}}$ below (4), and the fact that $\eta \leqslant 1$, one gets

$$
\left|(\Delta G)^{0}(\zeta, \eta)\right|_{\infty} \leqslant\left\|J_{G S}\right\|_{L_{\infty}}+\left(5+2 b_{2}\right)\|F\|_{L_{\infty}}=: M
$$

Assume that there a scalar exists $\kappa$ such that

$$
\kappa \geqslant\left(5+8\left|b_{2}\right|\right)|| C_{G} \|_{L_{\infty}}+2\left|H_{G}\right|_{\infty}+8\left|b_{2}\right|+4\left|H_{G S}\right|_{\infty}
$$

and the following inequality is true for a given rank $n \in \mathbb{N}$

$$
\left|(\Delta G)^{n}(\zeta, \eta)\right|_{\infty} \leqslant M \kappa^{n} \frac{\zeta^{n}+\eta^{n}}{n !}
$$

From (69), it follows

$$
\begin{aligned}
& \left|(\Delta G)^{n+1}(\zeta, \eta)\right|_{\infty} \leqslant\left\|C_{G}\right\|_{L_{\infty}} \int_{\eta}^{\zeta} \int_{0}^{\eta}\left|(\Delta G)^{n}(s, z)\right|_{\infty} d z d s \\
& +\left|H_{G}\right|_{\infty} \int_{0}^{\eta}\left|(\Delta G)^{n}(s, s)\right|_{\infty} d s \\
& \left.+2|| C_{G} \|_{L_{\infty}} \int_{0}^{\eta} \int_{0}^{s}\left|(\Delta G)^{n}(s, z)\right|_{\infty} d z d s\right] \\
& +4\left|b_{2}\right|\left\|C_{G}\right\|_{L_{\infty}} \int_{0}^{\eta} \int_{0}^{z} \int_{0}^{\mu}\left|(\Delta G)^{n}(\mu, s)\right|_{\infty} d s d \mu d z \\
& +4 b_{2} \int_{0}^{\eta}\left|(\Delta G)^{n}(z, 0)\right|_{\infty} d z+2\left|b_{2}\right| \int_{0}^{\eta}\left|(\Delta G)^{n}(z, z)\right|_{\infty} d z \\
& \left.+2\left|H_{G S}\right|_{\infty} \int_{0}^{\eta} \int_{0}^{z}\left|(\Delta G)^{n}(\mu, \mu)\right|_{\infty} d \mu d z\right]
\end{aligned}
$$

Consequently, using the recursive hypothesis (83), it holds

$$
\begin{aligned}
& \left|\Delta G^{n+1}(\zeta, \eta)\right|_{\infty} \leqslant \frac{M \kappa^{n}}{(n+1) !}\left(\| C _ { G } \| _ { L _ { \infty } } \left(\eta \zeta^{n+1}+\zeta \eta^{n+1}+4 \eta^{n+2}\right.\right. \\
& \left.\left.+8\left|b_{2}\right| \eta^{n+3}\right)+2\left|H_{G}\right|_{\infty} \eta^{n+1}+8\left|b_{2}\right| \eta^{n+1}+4\left|H_{G S}\right|_{\infty} \eta^{n+2}\right)
\end{aligned}
$$

From the fact that $\zeta, \eta \leqslant 1$, and (82), one concludes

$$
\left|(\Delta G)^{n+1}(\zeta, \eta)\right|_{\infty} \leqslant \frac{M \kappa^{n+1}}{(n+1) !}\left(\zeta^{n+1}+\eta^{n+1}\right)
$$

As the estimate (83) is proven for $n=0$ in (83), it follows by induction that $(\Delta G)^{n}$ exponentially converges and that the solution of (54)-(57) is given by (68).

Remark 1: It is worth pointing out that the upper bound we consider in the induction assumption (83) is not standard. Indeed [14], [15], among others, introduce the bound $\frac{(\zeta+\eta)^{n}}{n !}$, which cannot be used here. Indeed the integration of this bound for the specific boundaries (56)-(57) would cause the recursive method to fail. For example, in order to perform the iterative method, one needs to integrate $\int_{0}^{\eta} \int_{0}^{s}\left|(\Delta G)^{n}(z, s)\right|_{\infty} d z d s$ (which appear in (71) and in (84)). However, using the previous upper bound, one has

$$
\int_{0}^{\eta} \int_{0}^{s} \frac{(z+s)^{n}}{n !} d z d s=\left(2^{n+1}-1\right) \frac{\eta^{n+2}}{(n+2) !}
$$

The $2^{n+1}$ term is too large to be compatible with the recursive hypothesis. This is why, instead, we suggest to consider (83), which is a smaller bound.

\section{C. invertibility of the backstepping transformation}

Let us denote $\Pi$ the map that transforms the system (1)-(3) with $U(t)$ defined in (25) into the target system (6)-(8). It can be expressed as

$$
\Pi: \begin{aligned}
\mathrm{H} & \rightarrow \mathrm{H} \\
\left(q_{1}, q_{2}, q_{3}, q_{4}, q_{5}\right) & \mapsto\left(z_{1}, z_{2}, z_{3}, z_{4}, z_{5}\right)
\end{aligned}
$$

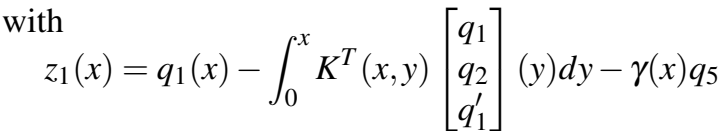

$$
\begin{aligned}
& z_{2}(x)=q_{2}(x)-\left[\begin{array}{lll}
q_{1}(x) & q_{2}(x) & q_{1}^{\prime}(x)
\end{array}\right] \Theta_{2} K(x, x) \\
& \left.-\int_{0}^{x}\left[K^{T}(x, y) \Theta_{1}-K_{y}^{T}(x, y) \Theta_{2}\right]\left[\begin{array}{l}
q_{1} \\
q_{2} \\
q_{1}^{\prime}
\end{array}\right](y)\right) d y \\
& +\left(k(x, 0)-\gamma(x) b_{3}\right) q_{4}+\left(m(x, 0)-\gamma(x) b_{1}\right) q_{5} \\
& z_{3}=q_{3}-\int_{0}^{1}\left[\begin{array}{lll}
q_{1} & q_{2} & q_{1}^{\prime}
\end{array}\right](y) K(x, y) d y-\gamma(1) q_{5} \\
& z_{4}=q_{4}, \quad z_{5}=q_{5}
\end{aligned}
$$

where $K$ is defined in (10) as the unique solution of (11)-(15), $\gamma$ in (16), and with $\Theta_{1}$ and $\Theta_{2}$ defined in (23).

Let us check that $z \in \mathrm{H}$. As $q \in \mathrm{H}$, using CauchySchwarz's inequality, it holds $z_{1} \in L_{2}(0,1)$. Similarly, one gets that $z_{1}^{\prime} \in L_{2}(0,1)$. Therefore $z_{1} \in H_{1}(0,1)$. Using CauchySchwarz's inequality on (90), one gets that $z_{2} \in L_{2}(0,1)$. The fact that $\left(z_{3}, z_{4}, z_{5}\right) \in \mathbb{R}^{3}$ is straightforward. Finally, one obtains that $z_{1}(1)=z_{3}$, as $q_{3}=q_{1}(1)$, and that $z_{1}(0)=z_{4}$ as $\gamma(0)=0$ and $q_{4}=q_{1}(0)$. Therefore $z \in \mathrm{H}$.

Note that by construction $\gamma(0)=0$ and $\gamma(x)=\frac{s(x, 0)}{b_{2}}$ which allows the backstepping transformation to map $\mathrm{H} \rightarrow \mathrm{H}$. Indeed, $\gamma(x)=\frac{s(x, 0)}{b_{2}}$ is a necessary condition for $z_{2}$ to be in $L_{2}(0,1)$. The former does not hold otherwise as a $z_{1}^{\prime}(0)$ term appears, which may be unbounded. Similarly $\gamma(0)=0$ is a necessary condition for $z_{5}$ to be in $\mathbb{R}$.

The existence of the inverse map $\Pi^{-1}$ can then be obtained by simply replacing $\lambda, \beta, a_{i}$ and $b_{i}$ with respectively $\lambda_{w}, \beta_{w}$, $c_{i}$ and $d_{i}$ in the previous analysis.

\section{Target system exponential stability}

Consider the target system defined in (6)-(8) and the following Lyapunov functional candidate

$$
\begin{aligned}
& V\left(w, w_{t}\right)=\int_{0}^{1}\left[\begin{array}{lll}
w & w_{t} & w_{x}
\end{array}\right] P_{0}\left[\begin{array}{lll}
w & w_{t} & w_{x}
\end{array}\right]^{T} \\
& +w(1) P_{1} w(1)+\left[\begin{array}{ll}
w(0) & w_{t}(0)
\end{array}\right] P_{2}\left[\begin{array}{c}
w(0) \\
w_{t}(0)
\end{array}\right]
\end{aligned}
$$

in which $P_{0} \in \mathbb{R}^{3 \times 3}, P_{1} \in \mathbb{R}$ and $P_{2} \in \mathbb{R}^{2 \times 2}$ are symmetric positive definite matrices. 
Lemma 3: For all $\lambda_{w}>0, \beta_{w}>0, c_{i}>0$, and $d_{i}>0$, there exist $P_{0}, P_{1}$ and $P_{2}$ symmetric positive definite matrices such that, it holds for a suitable $\rho>0$

$$
\dot{V}\left(w, w_{t}\right) \leqslant-\rho V\left(w, w_{t}\right)
$$

in which $V$ is defined in (93).

Proof of Lemma 3: Considering

$$
P_{0}=\left[\begin{array}{ccc}
\beta_{w} & \sigma & 0 \\
* & 1 & 0 \\
* & * & 1
\end{array}\right], \quad P_{1}=c_{2}, \quad P_{2}=\left[\begin{array}{cc}
\frac{d_{3}+\sigma d_{1}}{d_{2}} & \frac{\sigma}{d_{2}} \\
* & \frac{1}{d_{2}}
\end{array}\right]
$$

with

$$
0<\sigma<\min \left\{\sqrt{\beta_{w}}, \lambda_{w}, \frac{4 \beta_{w} \lambda_{w}}{\lambda_{w}^{2}+4 \beta_{w}}, \frac{4 c_{2}}{c_{1}}, d_{1}\right\}
$$

which is well-defined as $\beta_{w}, \lambda_{w}, c_{1}, c_{2}$, and $d_{1}$ are positive. With (96), the matrices $P_{0}$ and $P_{2}$ are positive definite. Computing the derivative of $V$ along the solution (93) leads to (6)-(8)

$$
\begin{gathered}
\dot{V}\left(w, w_{t}\right)=-\int_{0}^{1}\left[\begin{array}{lll}
w & w_{t} & w_{x}
\end{array}\right] Q_{0}\left[\begin{array}{lll}
w & w_{t} & w_{x}
\end{array}\right]^{T} \\
-\left[\begin{array}{ll}
w(1) & w_{t}(1)
\end{array}\right] Q_{1}\left[\begin{array}{c}
w(1) \\
w_{t}(1)
\end{array}\right]-\left[\begin{array}{ll}
w(0) & w_{t}(0)
\end{array}\right] Q_{2}\left[\begin{array}{c}
w(0) \\
w_{t}(0)
\end{array}\right]
\end{gathered}
$$

in which

$$
\begin{gathered}
Q_{0}=\left[\begin{array}{ccc}
2 \beta_{w} \sigma & \lambda_{w} \sigma & 0 \\
* & 2 \lambda_{w}-2 \sigma & 0 \\
* & * & 2 \sigma
\end{array}\right] \\
Q_{1}=\left[\begin{array}{cc}
2 c_{2} \sigma & c_{1} \sigma \\
* & 2 c_{1}
\end{array}\right], Q_{2}=\left[\begin{array}{cc}
\frac{2 \sigma d_{3}}{d_{2}} & 0 \\
* & \frac{2 d_{1}}{d_{2}}-\frac{2 \sigma}{d_{2}}
\end{array}\right]
\end{gathered}
$$

From (96) the matrices $Q_{0}, Q_{1}$, and $Q_{2}$, are positive definite. Therefore, (94) holds.

This Lyapunov functional candidate (93) is inspired by [14]. We have added the $P_{1}$ and $P_{2}$ terms to handle the dynamic boundaries under consideration.

\section{E. Stability in terms of $\Gamma$}

To conclude on the exponential stability of (1)-(3) under the control law (25), the equivalence between $V\left(w, w_{t}\right)$ in (93) and $\Gamma\left(u, u_{t}\right)$ in (27) is proven in the following lemma.

Lemma 4: There exist $\mu_{1}, \mu_{2}>0$ such that

$$
\begin{aligned}
& \Gamma\left(u, u_{t}\right) \leqslant \mu_{1} V\left(w, w_{t}\right) \\
& V\left(w, w_{t}\right) \leqslant \mu_{2} \Gamma\left(u, u_{t}\right)
\end{aligned}
$$

Proof : Only the establishment of (101) is detailed, as similar arguments hold for (100) using the inverse backstepping transformation (see Section III-C). From (9), (36), and (38), there exist $c_{1}, c_{2}, c_{3}, c_{4}>0$ such that

$$
\begin{aligned}
\|w\|_{L_{2}}^{2} & \leqslant c_{1}\left[\|u\|_{H_{1}}^{2}+\left\|u_{t}\right\|_{L_{2}}^{2}+u_{t}(0, t)^{2}\right] \\
w(1, t)^{2} & \leqslant c_{2}\left[\|u\|_{H_{1}}^{2}+\left\|u_{t}\right\|_{L_{2}}^{2}+u_{t}(0, t)^{2}+u(1, t)^{2}\right] \\
\left\|w_{t}\right\|_{L_{2}}^{2} & \leqslant c_{3}\left[\|u\|_{H_{1}}^{2}+\left\|u_{t}\right\|_{L_{2}}^{2}+u(0, t)^{2}+u_{t}(0, t)^{2}\right] \\
\left\|w_{x}\right\|_{L_{2}}^{2} & \leqslant c_{4}\left[\|u\|_{H_{1}}^{2}+\left\|u_{t}\right\|_{L_{2}}^{2}+u_{t}(0, t)^{2}\right]
\end{aligned}
$$

From (102)-(105) and the fact that $w(0, t)=u(0, t)$ and $w_{t}(0, t)=u_{t}(0, t) \quad($ as $\gamma(0)=0$ from (15)-(16)), one obtains (101).

\section{F. Final proof of Theorem 1}

Using Lemma 2, there exists a unique solution to (11)(16). From Lemma 1, this solution defines a backstepping transformation which maps the system (1)-(3) into (6)-(8) with the control law $U$ defined in (25). Moreover, from Lemma 3, one gets that (6)-(8) is exponentially stable if all its parameters are positive. Therefore, using Lemma 4, one establishes (28).

\section{CONCLUSION}

We have proposed a full-state feedback for a system where both the PDE and boundary conditions are unstable. In particular, we have generalized the control in [12], allowing a change of the in-domain viscous coefficient between the original and the target system. Our on-going works focus on the design of a collocated observer for the system (1)-(3).

A future direction of study could be to extend this design for a PDE such as $y_{t t}(x, t)=\left(a(x) y_{x}\right)_{x}(x, t)+\lambda(x) y_{t}(x, t)+$ $\beta(x) y(x, t)$ with two second order boundaries as considered in [1], [3], [11].

\section{REFERENCES}

[1] F. Conrad and A. Mifdal. Strong stability of a model of an overhead crane. Control and Cybernetics, 27(3):363-374, 1998.

[2] R. F. Curtain and H. Zwart. An introduction to infinite-dimensional linear systems theory, volume 21. Springer Science \& Business Media, 2012.

[3] F. Di Meglio and U. J. F. Aarsnes. A distributed parameter systems view of control problems in drilling. IFAC-PapersOnLine, 48(6):272278, 2015.

[4] C. G. Gal, G. R. Goldstein, and J. A. Goldstein. Oscillatory boundary conditions for acoustic wave equations, pages 623-635. Birkhäuser Vergal, Basel, 2004.

[5] K. F. Graff. Wave motion in elastic solids. Clarendon Press, Oxford, 1975.

[6] V. Keyantuo and M. Warma. The wave equation with Wentzell-Robin boundary conditions on Lp-spaces. Journal of Differential Equations, 229(2):680 - 697, 2006.

[7] M. Krstic and A. Smyshlyaev. Boundary Control of PDEs. SIAM Advances in Design and Control, 2008.

[8] F. D. Meglio, F. Bribiesca Argomedo, L. Hu, and M. Krstic. Stabilization of coupled linear heterodirectional hyperbolic PDE-ODE systems. Automatica, 87:281-289, Jan. 2018.

[9] T. Meurer and A. Kugi. Tracking control design for a wave equation with dynamic boundary conditions modeling a piezoelectric stack actuator. International Journal of Robust and Nonlinear Control, 21:542 - 562, 032011.

[10] S. Nicaise and C. Pignotti. Exponential stability of second-order evolution equations with structural damping and dynamic boundary delay feedback. IMA Journal of Mathematical Control and Information, 28(4):417-446, 2011.

[11] I. Peled, W. O'Connor, and Y. Halevi. On the relationship between wave based control, absolute vibration suppression and input shaping. Mechanical Systems and Signal Processing, 39(1):80 - 90, 2013.

[12] C. Roman, D. Bresch-Pietri, E. Cerpa, C. Prieur, and O. Sename. Backstepping observer based-control for an anti-damped boundary wave PDE in presence of in-domain viscous damping. In IEEE 55th Conference on Decision and Control, pages 549-554, Las Vegas, NV, Dec 2016.

[13] C. Sagert, F. Di Meglio, M. Krstic, and P. Rouchon. Backstepping and flatness approaches for stabilization of the stick-slip phenomenon for drilling. In IFAC Systems Structure and Control, pages 779-784, Grenoble, France, 2013.

[14] A. Smyshlyaev, E. Cerpa, and M. Krstic. Boundary stabilization of a 1-D wave equation with in-domain antidamping. SIAM Journal on Control and Optimization, 48(6):4014-4031, 2010.

[15] A. Smyshlyaev and M. Krstic. Closed-form boundary state feedbacks for a class of 1-D partial integro-differential equations. IEEE Transactions on Automatic Control, 49(12):2185-2202, Dec 2004.

[16] A. Smyshlyaev and M. Krstic. Boundary control of an anti-stable wave equation with anti-damping on the uncontrolled boundary. Systems \& Control Letters, 58(8):617-623, 2009. 